\title{
Characteristics of Autophagy Induced by Enterovirus71 (EV71) Viral Protein
}

\author{
Li Huan ${ }^{1,2}$, Gao Jinxue ${ }^{3}$, Liu Dan ${ }^{3}$, Long Hui ${ }^{1}$ and Wu Qingming ${ }^{2,3^{*}}$ \\ ${ }^{1}$ Department of Gastroenterology, Tianyou Hospital of Wuhan University of Science and Technology, Wuhan, Hubei, China \\ ${ }^{2}$ Hubei Province Key Laboratory of Occupational Hazard Identification and Control, Wuhan, Hubei, China \\ ${ }^{3}$ Medical School of Wuhan University of Science and Technology, Wuhan, Hubei, China
}

*Corresponding author: Wu Qingming, Medical School of Wuhan University of Science and Technology, Wuhan, Hubei, China, Tel: 086-13995601166; E-mail: wuhe9224@sina.com

Received date: September 15, 2018; Accepted date: October 08, 2018; Published date: October 14, 2018

Copyright: @ 2018 Huan L, et al. This is an open-access article distributed under the terms of the Creative Commons Attribution License; which permits unrestricted use; distribution; and reproduction in any medium; provided the original author and source are credited.

\begin{abstract}
Hand, Foot and Mouth Disease (HFMD) is a type of infectious disease that is more commonly seen in children under the age of 6 years. It was first reported in New Zealand in 1957, and then outbreaks occurred in Europe, America and Asia. In recent years, the incidence of HFMD in China is also on the rise. HFMD was caused by Coxsackievirus A16 (CVA16) and Human Enterovirus 71 (EV71) and other virus. CVA16 usually causes mild cases and is less harmful, while EV71 infection causes not only mild cases, but also severe and fatal cases; it has become a cause of concern. The mechanism of HFMD caused by EV71 virus is not completely clear. After it infects host cells, the EV71 virus genome undergoes replication, translation, assembly, and the release of virosomes. Some studies have reported that EV71 could induce host cell autophagy. Autophagy is a cellular process necessary for the lysosomal degradation and recycling of proteins and entire organelles. It can act as not only a defense mechanism to prevent environmental damage to cells, also induce cell death in eukaryotes. To explore the correlation and characteristics of EV71 virus, EV71 viral protein and autophagy, we used EV71 and recombinant plasmid pcDNA3.1 $(+)$-HA-X to infect human 293T cells, and we found that autophagy could be induced by EV71. With the prolongation of infection, autophagy presents a dynamic trend. And the protein that causes autophagy is structural protein VP1 and non- structural protein 2A.
\end{abstract}

Keywords: Enterovirus71; Viral protein; Autophagy; Microtubuleassociated protein light chain 3(LC3); Protein 62(p62)

\section{Introduction}

Enterovirus71 (EV71) belongs to the Enterovirus family and has neurotoxicity, which is one of the primary viruses causing severe viral infection diseases such as hand-foot-mouth disease [1-3]. The EV71 genome encodes a polyprotein that could be hydrolyzed by both host and viral proteases to produce four structural proteins (VP1, VP2, VP3, VP4) and seven non-structural proteins (2A, 2B, 2C, 3A, 3B, 3C and 3D)[4]. Amino acid polymorphism at VP1-145 is involved in EV71 replication and in vivo fitness [5]. The virulence of the viruses with glutamic acid (E) at VP1-145 (VP1-145E) is stronger than those with glutamine $(\mathrm{Q})$ or glycine $(\mathrm{G})$ at VP1-145 (VP1-145Q/G) [6]. Currently, the mechanism of EV71-induced neural system infection is not clear. Studies suggest the autophagy process induced by EV71 is related to its ability in apoptosis and necrosiss [7].

Autophagy is classified as type II programmed cell death, including macroautophagy, microautophagy, and Chaperone-Mediated Autophagy (CMA). The progress of autophagy includes the formation of autophagic precursor, autophagosome, autophagy lysosome, and autophagy lysosomes degradation [8]. In this process, the esterification of microtubule associated protein light chain 3 (LC3) is an important marker of autophagosome formation. The degradation of the p62, which functions in linking LC3 and ubiquitination, is a hallmark of autophagy. Autophagy plays an important role in preventing infection and promoting immunity. Additionally, autophagy is capable of degrading invasive microorganisms and eliminating pathogen-infected cells. On the other hand, some pathogens can induce autophagy and promote viral replication. Studies have reported that EV71 virus can induce autophagy in human rhabdomyosarcoma (RD) and neuroblastoma (SK-N-SH) cells [7,9].

Our study will investigate whether EV71 can induce autophagy in 293T cells and identify EV71 viral proteins involved in autophagy. The in-depth exploration of the interactive mechanism between viral proteins and cellular autophagy will provide the experimental evidence for the pathogenic mechanism of EV71-related antiviral immunity.

\section{Materials and Methods}

\section{Reagents and materials}

Minimum essential medium, Dulbecco's modified Eagle's medium, and TRIzo were purchased from Invitrogen Corporation (Carlsbad, CA, USA). Plasmid Mini Kit I and Gel Extraction Kit were purchased from Omega Bio-tek (Atlanta, GA, USA). Tryptone, Yeast extract were purchased from Oxoid (Basingstoke, Hampshire, UK). Agar, Tris-base, 3-Methyladenine (3-MA), rapamycin, RNase Inhibitor, anti-LC3 antibody produced in rabbit and anti-p62 antibody produced in rabbit were purchased from Sigma-Aldrich (St Louis, MO, USA). Goat antimouse IgG $\mathrm{H}$ and L (HRP), goat anti-rabbit IgG, alexa fluor 555labeled donkey anti-mouse IgG, Anti-HA tag antibody were purchased from Abcam Cambridge, UK. $\beta$-actin mouse monoclonal antibody was purchased from ProteintechRosemont, IL, USA. Prestained protein marker and ECL chemiluminescent substrate reagent kit were 
purchased from Thermo Fisher Scientific Waltham, MA, USA. M-MLV and T4-DNA Ligase were purchased from Promega (Madison, WI, USA). KOD-plus neo was purchased from ToyoboOsaka, Japan. Taq 2X Master Mix was purchased from NovoGeneBeijing, China. Human rhabdomyosarcoma cellsRD cells) and 293T cells were kindly provided by Wuhan Institute of Virology, Chinese Academy of Sciences Wuhan, Hubei, China).

\section{RD and 293T cells}

$\mathrm{RD}$ cells and $293 \mathrm{~T}$ cells were cultured in DMEM medium containing $10 \%$ fetal bovine serum, $100 \mathrm{U}$ penicillin and $100 \mathrm{mg} / \mathrm{ml}$ streptomycin at $37^{\circ} \mathrm{C}$ with $5 \% \mathrm{CO}_{2}$.

\section{Effect of EV71 virus on 293T cells autophagy}

EV71 virus amplification: $\mathrm{RD}$ cells were maintained in L-glutamine containing Dulbecco's modified Eagle's medium (DMEM) supplemented with Foetal Bovine Serum (FBS). At $70-80 \%$ of confluence, EV71 virus supernatants were applied on the bottom layer of the cells and the cells were cultured at $37^{\circ} \mathrm{C}$ in the $5 \% \mathrm{CO}_{2}$ incubator for 2 hours. Then, $4 \mathrm{ml}$ of maintenance solution containing 2\% FBS was added into the culture and maintained for 24 hours. The virus supernatant was collected and stored at $-80^{\circ} \mathrm{C}$ until use.

Virulence assay of EV71: The end point dilution method was utilized to determine the virulence through measuring 50\% Tissue Culture Infectious Dose (TCID 50). The TCID 50 was calculated by Reed-Muench method. Under the sterile condition, RD cells were digested with trypsin containing DMEM $+2 \%$ FBS. The cells were dissected and the cell density was counted using the hemocytometer. After the cell density was adjusted to $2 \times 10^{5}$ cells $/ \mathrm{mL}$ with DMEM $+2 \%$ FBS, $100 \mu \mathrm{L}$ of cell culture media $\left(2 \times 10^{4}\right.$ cells/well $)$ were added into each well of the 96-well plate. The collected viral supernatants were serially diluted (10-fold in succession), from $10^{-1}$ to $10^{-10}$ with DMEM $+2 \%$ FBS. $100 \mu \mathrm{L}$ diluted virus suspension was added into the cell well and a total of 8 wells in a tandem row were inoculated. Control cells were set up at the left and right column of the 96-well plate with only $100 \mu \mathrm{L} \mathrm{DMEM}+2 \% \mathrm{FBS}$ added into each well. The culture plates were placed in the $37^{\circ} \mathrm{C}, 5 \% \mathrm{CO}_{2}$ incubator, and the morphology of the cells were observed daily under an inverted microscope for consecutive 7 days. TCID 50 was calculated based on Cytopathic Effect (CPE).

In Vitro cell transfection: 293 T cells were seeded onto $35 \mathrm{~mm}$ plates and incubated for 20 hours when its convergence was up to $30 \%-40 \%$. The cells were transfected with LC3-EGFP plasmid using the calcium phosphate method. After incubation for 8 hours, the transfection efficiency was analysed at indicated time (16-48 hours).

Drug intervention experimental groups: Experimental groups included the autophagy enhancement group $(1 \mu \mathrm{M}$ rapamycin $+5 \mathrm{moi}$ EV71), autophagy inhibition group (1mM 3-MA+5 moi EV71), autophagy group (5 moi EV71) and the control group (without EV71 infection).

Western blotting: Western Blotting was performed at time points of $0 \mathrm{~h}, 4 \mathrm{~h}, 8 \mathrm{~h}, 16 \mathrm{~h}$ and $32 \mathrm{~h}$. The proteins were extracted from NP40 cell lysates and then electrophoresed on PVDF membrane in SDS-PAGE. The activated membrane was immersed in electrophoretic transfer membrane $(25 \mathrm{mmol} / \mathrm{L}$ of Tris at $\mathrm{pH} 8.3,192 \mathrm{mmol} / \mathrm{L}$ of glycine, and $20 \%$ methanol) for $10 \mathrm{~min}$. $10 \mathrm{~mL}$ of 1:2000 diluted primary antibody (primary anti-HAtag multi-antibody $5 \mu \mathrm{L}+10 \mathrm{~mL}$ of TBS) was added into the medium and hybridized for $2 \mathrm{~h}$ at $37^{\circ} \mathrm{C}$. After incubated with the primary antibody, the membrane was cleaned for three times with TBST, once for 10 minutes. The horseradish-conjugated secondary antibody (1:5000 goat anti-mouse IgG-HRP) was added and incubated at $37^{\circ} \mathrm{C}$ for $1 \mathrm{~h}$. After the excessive antibodies were washed out, the same volume of ECL luminescence reagents A and B were mixed and reacted with the dried membrane for 1 minute after the membrane was exposed in the dark room; image software was used for gray analysis of the bands.

Autophagy assay of immunofluorescence cell: The LC3-EGFP plasmid was transfected into $293 \mathrm{~T}$ cells. After 24-hour cultivation, cells were fixed with paraformaldehyde at room temperature for 15 minutes, permeabilized with $0.2 \%$ Triton X-100 for $15 \mathrm{~min}$, and blocked with $5 \% \mathrm{BSA}$ at $37^{\circ} \mathrm{C}$ for $2 \mathrm{~h}$. After incubated with the primary antibody at $4^{\circ} \mathrm{C}$ overnight, the secondary antibody for $40 \mathrm{~min}$, and stained with DAPI for $15 \mathrm{~min}$, autophagy was observed through fluorescence microscopy.

\section{Effect of EV71 protein on autophagy}

Construction of the eukaryotic expression vector of EV71: The EV71 virus RNAs were extracted and RT-PCR was performed to obtain viral cDNAs. According to the sequence of the EV71 type-XF strain (GenBank: JQ804832), the upstream and downstream primers of each structural protein and non-structural protein were designed (primers synthesized by Beijing Qingke Biotechnology Co., Ltd.). With the 11 pairs of primers, cDNAs were used as the template to amplify viral genes of VP1, VP2, VP3, VP4, 2A, 2B, 2C, 3A, 3AB, 3C, 3D and construct 11 recombinant plasmids of pcDNA3.1 (+)-HA-VP1, pcDNA3.1 (+) -HA-VP2, pcDNA3.1(+)-HA-VP3, pcDNA3.1(+)-HAVP4, pcDNA3.1(+)-HA-2A, pcDNA3.1(+)-HA-2B, pcDNA3.1(+)HA-2C, pcDNA3.1(+)-HA-3A, pcDNA3.1(+) -HA-3AB, pcDNA3.1(+)-HA-3C, pcDNA3.1(+)-HA-3D. After PCR product gels were recovered, 11 target genes and PCDNA3.1 $(+)$ vectors were digested. Then the target genes and linearized vectors were ligated. Competent cells were prepared and the ligated products were electroporated into E. coli. Small amount of DNAs were extracted from the recombinant plasmids for confirmation via PCR (denaturation at $94^{\circ} \mathrm{C}$ for 5 minutes, predenaturation at $94^{\circ} \mathrm{C}$ for 30 seconds, annealing at $59^{\circ} \mathrm{C}$ for $30 \mathrm{sec}$, extension at $72^{\circ} \mathrm{C}$ for 1 minute, for totally 29 cycles from the second step, and final extension at $72^{\circ} \mathrm{C}$ for $10 \mathrm{~min}$ ) (Figure S1). Recombinant plasmid was sequenced by Beijing Qingke Biotechnology Co., Ltd (Figure S2).

Expression of recombinant viral proteins in 293T cells: The 293T cells were transfected with 11 recombinant plasmid using the calcium phosphate method and the 293T cells transfected with pcDNA3.1 (+) empty carrier were set as the control. Viral protein expression in 293T cells was detected by Western Blotting.

The LC3-EGFP and pcDNA3.1-HA-X co-expression plasmids were co-transfected into $293 \mathrm{~T}$ cells using the same method as above.

\section{Statistical analysis}

SPSS19.0 statistical software was used for statistical analysis. Mean \pm $\mathrm{SD}$ was used to analyse quantitative data. Paired t-test was used to perform group comparison. $\mathrm{P}<0.05$ was considered statistically significant. 
Citation: Huan L, Jinxue G, Dan L, Hui L, Qingming W (2018) Characteristics of Autophagy Induced by Enterovirus71 (EV71) Viral Protein. J Trop Dis 6: 286. doi:10.4172/2329-891X.1000286

Page 3 of 7

\section{Results}

Green fluorescence was detected in EV71-infected $293 \mathrm{~T}$ cells transfected with pEGFP-LC3 plasmid (Figure 1). For the four groups of cells, the number of fluorescent spots was $2.67 \pm 0.58,36.33 \pm 3.05$, $34.00 \pm 1.00$, and $4.67 \pm 0.58$, respectively. Inhibitor 3-Methyladenine (3-MA) decreased the number of fluorescent spotsindicating autophagy inhibition. While Rapamycin increased the number of fluorescent spots indicating autophagy induction (Figure 2).

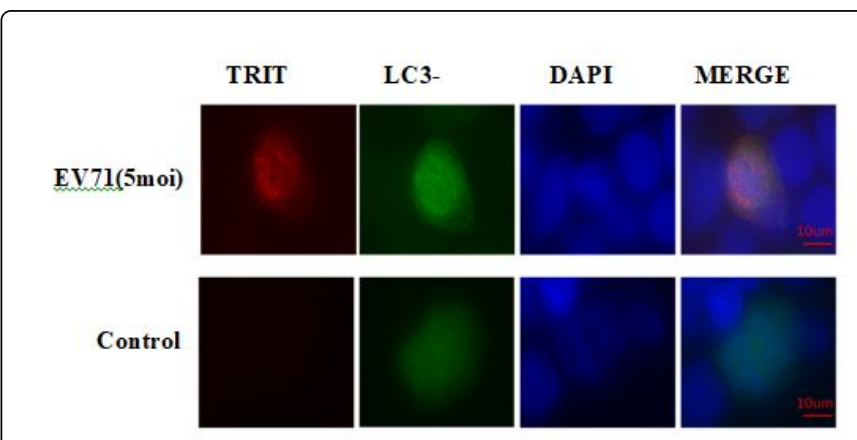

Firgure 1: The expression of LC3 was detected by immunofluorescence assay. The Enterovirus 71 was orange red stained. The LC3-EGFP plasmid was green stained. The nucleus was blue-stained by DAPI. MERGE was the three fusion image of TRITC, LC3-EGFP and DAPI. The images were captured at 60 $\times$ magnification. Scale bar denotes $10 \mu \mathrm{m}$.

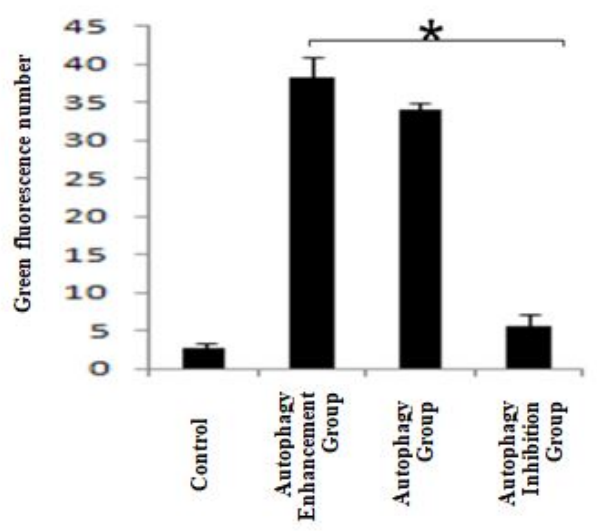

Firgue 2: The green fluorescence number of 293T cells was detected by immunofluorescence assay. Data are presented as the mean \pm SD. ${ }^{*} \mathrm{p}<0.05$ versus each group. The green fluorescence number of each group was $2.67 \pm 0.58,36.33 \pm 3.05,34.00 \pm 1.00,4.67 \pm 0.58$.

At different time points $(0 \mathrm{~h}, 4 \mathrm{~h}, 8 \mathrm{~h}, 16 \mathrm{~h}$ and $32 \mathrm{~h})$, the expression level of P62 was significantly different $(\mathrm{P}<0.05)$ (Figures 3 and 4$)$. The levels of p62 in each time points were $0.64 \pm 0.03 \mathrm{mg} / \mathrm{L}, 0.40 \pm 0.03$ $\mathrm{mg} / \mathrm{L}, 0.15 \pm 0.01 \mathrm{mg} / \mathrm{L}, 0.61 \pm 0.01 \mathrm{mg} / \mathrm{L}, 0.20 \pm 0.02 \mathrm{mg} / \mathrm{L}$. With the increase of EV71 infection duration, the expression of p62 firstly decreased then increased, but decreased again at last.

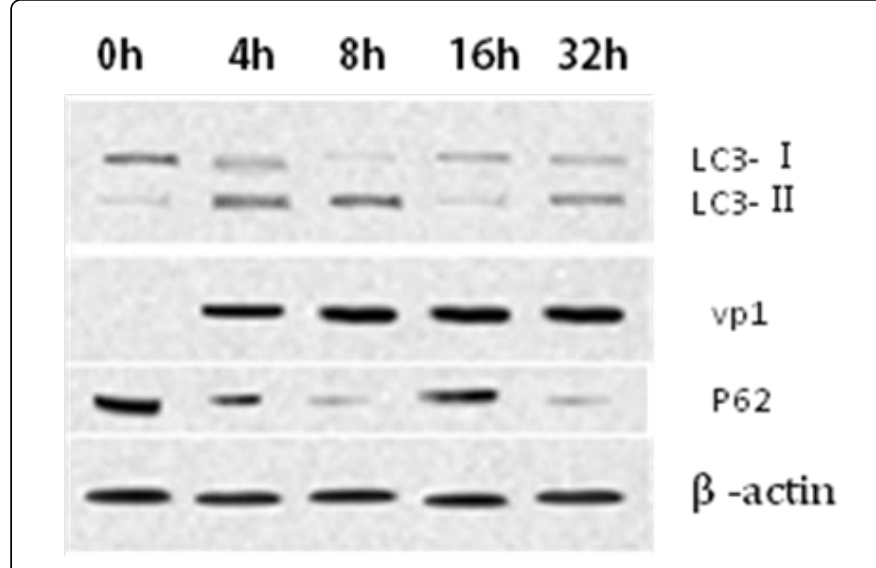

Figure 3: After EV71 infection for different time (0h, 4h, 8h, 16h and 32h), the expression levels LC3-, LC3-, VP1, p62 were measured by western blot, and $\beta$-actin served as the loading control.

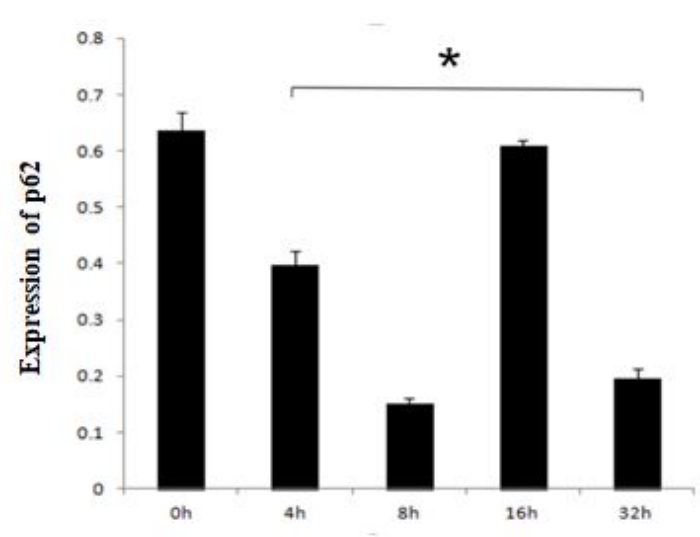

Figure 4: Expression of p62 at different time points. Data were presented as the mean $\pm \mathrm{SD}(\mathrm{n}=3),{ }^{*} \mathrm{p}<0.05$ at different time points, there were significant different between each two groups. With the increase of EV71 infection duration, the expression of p62 firstly decreased then increased, but decreased again at last.

The amount of LC3-II increased at $4 \mathrm{~h}$ following infection, reached to maximum at $8 \mathrm{~h}$, and then started decreasing from $8 \mathrm{~h}$, reached minimum at $16 \mathrm{~h}$, but began to rise again after $16 \mathrm{~h}$ (Figures 3,5 and 6 ). It suggests that the expression of LC3-II was negatively correlated with the expression of P62. 
Citation: Huan L, Jinxue G, Dan L, Hui L, Qingming W (2018) Characteristics of Autophagy Induced by Enterovirus71 (EV71) Viral Protein. J
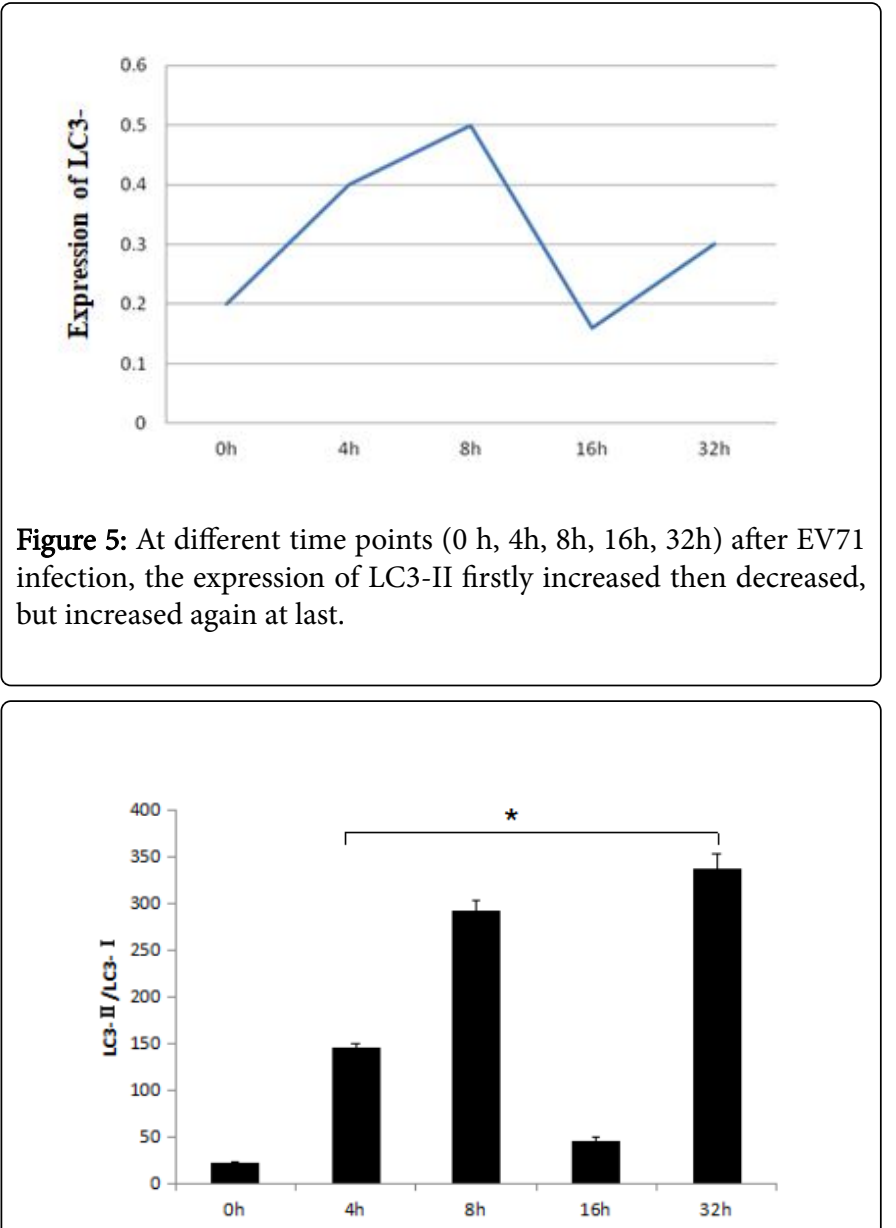

Figure 6: Ratio of LC3-to LC3- at different time points. Data were presented as the mean $\pm \mathrm{SD}(\mathrm{n}=3),{ }^{*} \mathrm{p}<0.05$ at different time points, there were significant different between each two groups. With the increase of EV71 infection duration, the ratio of LC3-to LC3increased firstly then decreased, but increased again at last.

\section{Viral protein-induced autophagy}

11 recombinant plasmids were successfully transfected, and the viral proteins were all expressed (Figure 7). In 11 viral protein-infected 293T cells, specific fluorescence was only observed in cells transfected with VP1 and 2A, suggesting VP1 and 2A could induce the formation of autophagosomes (Figure 8).
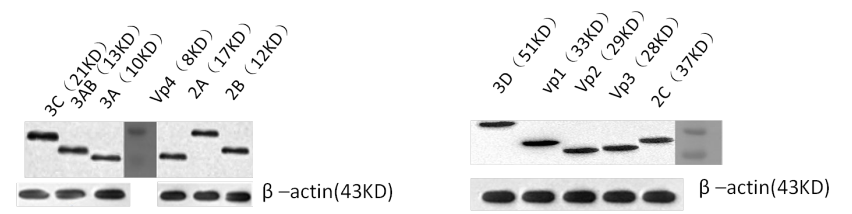

Figure 7: Western blotting for recombinant viral protein expression in $293 \mathrm{~T}$ cells. The molecular weights of VP1, VP2, VP3, VP4, 2A, $2 \mathrm{~B}, 2 \mathrm{C}, 3 \mathrm{~A}, 3 \mathrm{AB}$, and $3 \mathrm{D}$ were $33 \mathrm{kDa}, 28 \mathrm{kDa}, 27 \mathrm{kDa}, 8 \mathrm{kDa}, 17$ $\mathrm{kDa}, 12 \mathrm{kDa}, 37 \mathrm{kDa}, 10 \mathrm{kDa}, 13 \mathrm{kDa}, 21 \mathrm{kDa}, 21 \mathrm{kDa}, 51 \mathrm{kDa}$.

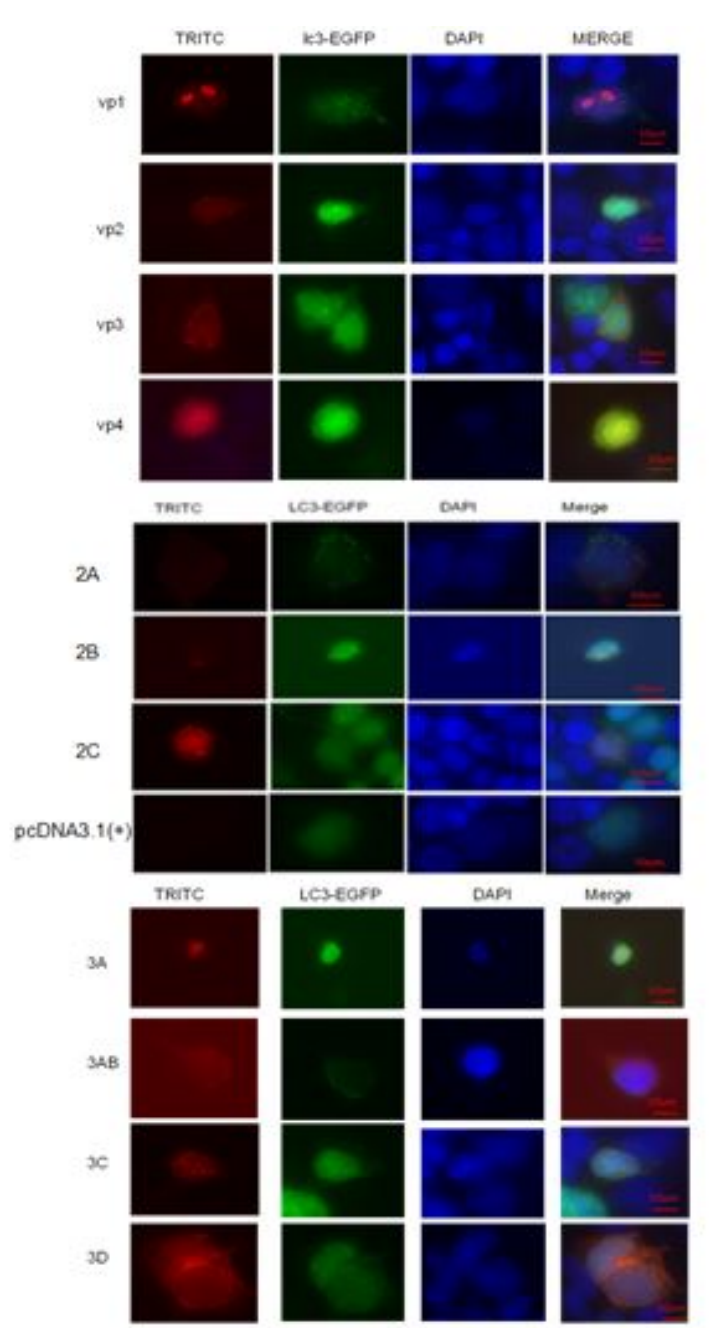

Figure 8: Immunofluorescence studies in 293T cells infected with 11 viral proteins. pcDNA3.1(+) served as the negative control. Specific fluorescence was only observed in cells transfected with VP1 and 2A. The EV71 was orange red stained. The LC3-EGFP plasmid was green stained. The nucleus was blue-stained by DAPI. MERGE was the three fusion image of TRITC, LC3-EGFP and DAPI. The images were captured at $60 \times$ magnification. Scale bar denotes $10 \mu \mathrm{m}$.

Western blotting results showed that p62, LC3-II, and LC3-II/LC3-I had significant expression changes in VP1 and 2A groups, but not in other groups, further indicating VP1 and 2A could induce autophagy (Figures 9, 10 and 11). 

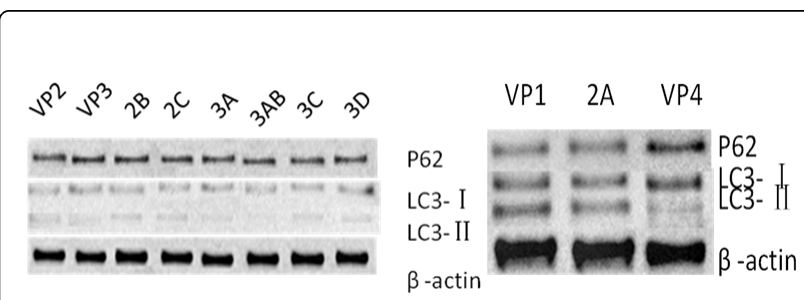

Figure 9: Western blotting studies for the expression of p62, LC3-, LC3-in 11 viral protein-infected $293 \mathrm{~T}$ cells. $\beta$-actin served as the loading control. The expression of P62 decreased in VP1 and 2A groups, while the expression of LC3-II, and LC3-II/LC3-I increased in the same groups.

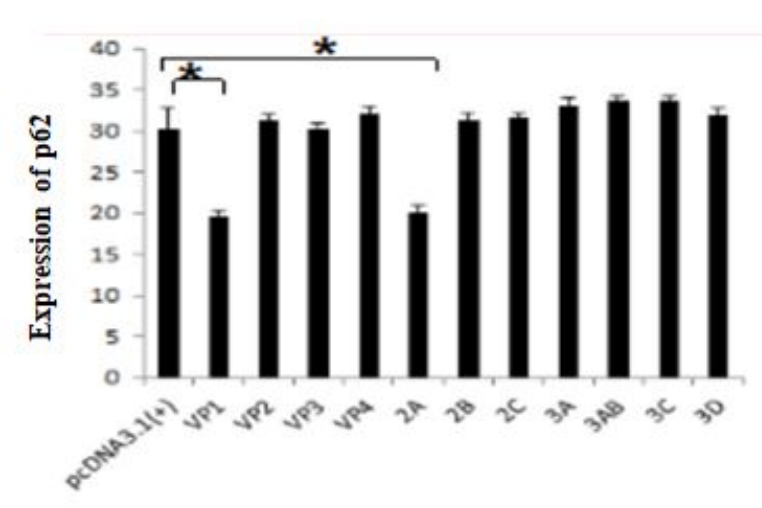

Figure 10: Expression of p62 in 12 groups, including 11 viral protein-infected groups and pcDNA3.1 (+) negative control group. Data were presented as the mean $\pm \mathrm{SD}(\mathrm{n}=3)$, ${ }^{*} \mathrm{p}<0.05$ versus each group. The expression of $\mathrm{p} 62$ decreased in VP1 and $2 \mathrm{~A}$ groups, but not in other groups.

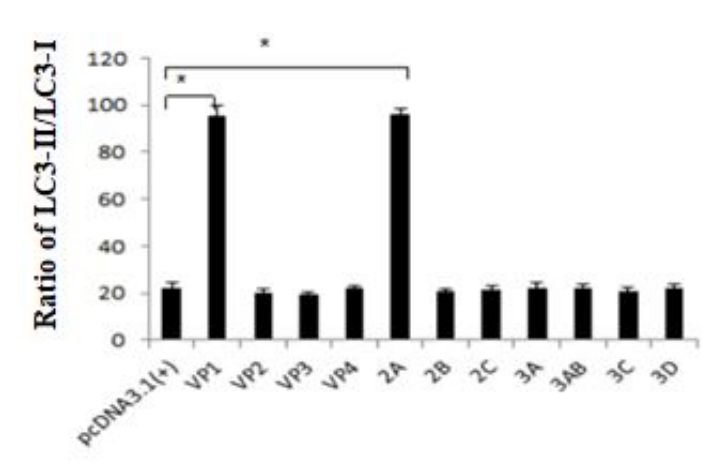

Figure 11: Ratio of LC3- to LC3-in 12 groups, including 11 viral protein-infected groups and pcDNA3.1 (+) negative control group. Data were presented as the mean $\pm \mathrm{SD}(\mathrm{n}=3),{ }^{*} \mathrm{p}<0.05$ versus each group. With the increase of EV71 infection duration, the ratio of LC3-II to LC3-I increased in VP1 and 2A groups, but not in other groups.

\section{Discussion}

Hand, Foot and Mouth Disease (HFMD) is a type of infectious disease that is more commonly seen in children under the age of 6 years [10]. According the epidemiological report for the period of 2008-2015 in China, HFMD showed an increasing trend in China [3]. Most patients have mild symptoms and self-limiting, but some patients may have severe complications such as meningitis, encephalitis, myelitis, encephalomyelitis, myocarditis, and neurogenic pulmonary edema, resulting in high mortality and morbidity $[11,12]$. Pathogens causing HFMD are human Enteroviruses, including EV71, Coxsackievirus (CV), and Non-EV-A71/CV-A16 HEVs [13,14]. Among these pathogens, EV71 and CV A group 16 are primary pathogens. It's reported that EV71 infection significantly increased the risk of severe complications and death $[3,12,15,16]$. EV71 is a single positive-stranded RNA virus with no envelopes or projections. After it infects host cells, the virus genome undergoes replication, translation, assembly, and the release of virosomes. Some studies have reported that EV71 could induce host cell autophagy [17]. Lee and colleagues revealed that EV71 infection in suckling mice induced an amphisome formation accompanied, by the autophagic flux in the brain tissues [9]. Too and colleagues revealed that autophagy was remarkably upregulated in EV71-infected NSC-34 cells [18].

Autophagy is a cellular process necessary for the lysosomal degradation and recycling of proteins and entire organelles. It can act as not only a defense mechanism to prevent environmental damage to cells also induces cell death in eukaryotes. The regulation of autophagy is achieved through ubiquitin-like protein systems [19], mammalian target of rapamycin (mTOR) [20], acetylated protein systems [21], methylation [22], microRNA (miRNA) [23], and transcription factors [24]. Autophagy-related genes (ATGs) play the key role in this process [8]. For example, autophagy can be induced by PI3K core complex which contains Beclin1/BECN1 (ATG6), ATG9, TAG14L, Ultraviolet Radiation Resistance-associated Gene Protein (UVRAG) and other proteins [25]. The extension of autophagosome membrane involves two ubiquitin-like proteins (ATG12 and ATG8/LC3). After LC3 is synthesized, with the catalysis of ATG4 and ATG7, LC3-I combines with Phosphatidylethanolamine (PE) on the surface of autophagic membrane to form LC3-II, which is the key step in autophagosome membrane fusion. As a result, the volume of autophagosome is constantly expanding. The ubiquitin protein p62 is a key protein in the degradation of the ubiquitinated proteasome system and the autophagy-lysosome system. During the formation of autophagosomes, p62 is able to specifically recognize the LC3 protein through the ubiquitin signalling pathway, connecting the ubiquitinated LC3 protein to the damaged mitochondria, facilitating the transportation and ultimate degradation by lysosomes. Thus LC3 and p62 are reliable markers for the level of autophagy. And the p62 level can be used as an indicator for the process of autophagic flow.

Studies reported that some viruses, including polio virus [26], hepatitis C virus [27,28], dengue virus $[29,30]$ can induce autophagy. Coxsackie virus can either inhibit autophagy or induce the formation of autophagosomes to increase viral replication [31,32]. $\mathrm{Xi}$ and the colleagues determined autophagy was induced by EV71 virus in RD-A cells, with an increase in the time course of infection $(6 \mathrm{~h}, 9 \mathrm{~h}, 12 \mathrm{~h}$ and 24h) by EV71, the conversion of LC3-I to LC3-II and the degradation of p62 increased accordingly [7]. Fu and the colleagues [33] found that EV71 could promote viral replication and induce autophagy through regulating the expression of has-miR-30a. Other studies reported EV71 
mediated VP1-induced autophagy through the endoplasmic reticulum stress pathway [34], and autophagy inhibitors could inhibit the release of EV71 virus [7].

In our study, we found that the infection of EV71 into 293T cells caused significant increase of the fluorescence spots. The addition of autophagy inhibitors significantly decreased the fluorescence spots. Interestingly, if agonists were added, the fluorescence spots increased again. However, there was no change of pEGFP-LC3 fluorescence spots observed in the control group. Our results suggest that EV71 could induce autophagy.

There were two types of conclusions on the expression trend of LC3II after EV71 virus infection, some studies suggested that the expression trend was increasing which was time-and dose-dependent $[7,35]$. In addition, other studies suggested that the expression of LC3 II did not show a continuous increasing trend, it increased first, then decreased [9,36]. In our study, we also demonstrated that enterovirus71 infection could induce the elevation of LC3-II expression in the early phase. In the middle phase, LC3-II returned to baseline level, with a down-regulation trend. However, in the late phase, LC3-II level increased again. It suggested that the process of autophagy was changing during the EV71 infection, displaying the autophagy flow and dynamic autophagy. This conclusion was similar to the experimental result of $\mathrm{Xi}$, the expression level of LC3-II was upregulated and the expression level of p62 was down regulated at the end of study [7], but we also found that the change of LC3-II and p62 was a dynamic process rather than a single trend change. In addition, in the study of SK-N-SH cells infected with EV71, Lee and the colleagues found that the expression level of LC3-II was gradually increased and reached the peak at $9 \mathrm{~h}$ p.i., as the time of autophagy prolonged, the expression level of LC3-II was then decreased after $12 \mathrm{~h}$ p.i. [9]. In our study, the expression level of LC3-II reached the peak at $8 \mathrm{~h}$ p.i., and then decreased to the lowest level after $16 \mathrm{~h}$ p.i., this dynamic process was similar to the result of Lee. However, when the observation time was extended to $32 \mathrm{~h}$, the expression level of LC3-II was showed an upward trend eventually. In our research, the dynamic trends of LC3-II and p62 was different from those mentioned earlier? This change has not been reported in previous studies. The specific reason was unknown, it was may be related to the terminal observing time which was inconsistent in the two studies. Moreover, the reason for these dynamic changes was not very clear. In recent study, Chinmoy Sarkar and his colleagues reported that they found a timedependent increase in the levels of LC3-II, which peaked between 1 and 3 days after injury and then gradually decreased by $\mathrm{d}$ 7; it may be caused by lysosomal dysfunction [36]. This may be the reason, but it is needed further investigation to clarify.

Virus infection could induce autophagy, and many viral proteins can induce autophagy also [37-40]. A recent study showed that neither $2 \mathrm{~B}$, $2 \mathrm{C}, 3 \mathrm{~A}, 3 \mathrm{~B}, 3 \mathrm{AB}, 3 \mathrm{C}$ nor $3 \mathrm{D}$ could trigger autophagy individually [7]. Another research had revealed that overexpression of the nonstructural protein $2 \mathrm{BC}$ of EV-A71 induced autolysosome formation to facilitate its replication. Therefore, the relationship between EV71 virus protein and autophagy is not yet clear [41]. In order to clarify whether EV71 virus protein could induce autophagy, and identify the protein type, we used genetic engineering method to transfect $293 \mathrm{~T}$ cells with 11 EV1-encoded proteins, including 4 structural proteins and 7 nonstructural proteins. We found that only VP1 and $2 \mathrm{~A}$ proteins were able to cause autophagy. These results suggest that the EV71-induced autophagy could be caused by VP1 and/or $2 \mathrm{~A}$, but not the entire virion. However, the specific mechanism is not clear yet. As one of the structural proteins of the EV71 virus, VP1 has the function of helper virus infection and joint cell receptor. At present, the study of VP1 tends to focus on its construction, mutation of different gene loci and immunogenicity [42-44], there is few studies on its pathogenesis, we may find out the pathway of VP1 induced autophagy through the study of VP1 immunogenicity and autophagy related receptors. The $2 \mathrm{~A}$ protein of EV71 acts on the replication of virus and the process of immune escape [45,46]. Perhaps, the study of 2A-autophagy and immune escape can identify the autophagy pathway caused by protein $2 \mathrm{~A}$.

In conclusion, in intro study suggests that the process of autophagy induced by EV71 is dynamic, and the occurrence of autophagy was related to EV71 structural protein VP1 and/or non-structural protein 2A. This may be helpful for the observation and treatment of HFMD. But, there are some questions left, the reason of autophagy dynamic process and the autophagy mechanism caused by protein VP1 and/or $2 \mathrm{~A}$ are not clear. It needs to be further discussed.

\section{References}

1. Sarma N, Chakraborty S, Dutta A, Sadhukhan PC (2017) Hand, foot and mouth disease in West Bengal, India: A preliminary report on Clinicovirological trend over 3 successive years (2013-2015). Indian J Dermatol 62: 486-490.

2. Puenpa J, Auphimai C, Korkong S, Vongpunsawad S, Poovorawan Y (2018) Enterovirus A71 infection, Thailand, 2017. Emerg Infect Dis 24: 1386-1387.

3. Huang J, Liao Q, Ooi MH, Cowling BJ, Chang Z,et al. (2018) Epidemiology of recurrent hand, foot and mouth disease, China. 2008-2015. Emerg Infect Dis 24(3):432-442.

4. Han L, Li K, Jin C, Wang J, Li Q, et al. (2017) Human enterovirus 71 protein interaction network prompts antiviral drug repositioning. Sci Rep 7: 43143 .

5. Kataoka C, Suzuki T, Kotani O, Iwata-Yoshikawa N, Nagata N, et al. (2015) The role of vp1 amino acid residue 145 of Enterovirus 71 in viral fitness and pathogenesis in a cynomolgus monkey model. PLoS Pathog 11: e1005033.

6. Fujii K, Sudaka Y, Takashino A, Kobayashi K, Kataoka C, et al. (2018) VP1 amino acid residue 145 of enterovirus 71 is a key residue for its receptor attachment and resistance to neutralizing antibody during cynomolgus monkey infection. J Virol 92.

7. Xi X, Zhang X, Wang B, Wang T, Wang J, et al. (2013) The interplays between autophagy and apoptosis induced by enterovirus 71. PLoS One 8: e56966.

8. Parzych KR, Klionsky DJ (2014) An overview of autophagy: morphology, mechanism, and regulation. Antioxid Redox Signal 20: 460-473.

9. Lee YR, Wang PS, Wang JR, Liu HS (2014) Enterovirus 71-induced autophagy increases viral replication and pathogenesis in a suckling mouse model. J Biomed Sci 21: 80.

10. Koh WM, Bogich T, Siegel K, Jin J, Chong EY, et al. (2016) The epidemiology of hand, foot and mouth disease in Asia: A systematic review and analysis. Pediatr Infect Dis J 35: e285-e300.

11. Yang ZY, Chen XQ, Sun D, Wei D (2018) Mortality in children with severe hand, foot and mouth disease in guangxi, China. Indian Pediatr 55: 137-139.

12. Koh WM, Badaruddin H, La H, Chen MI, Cook AR (2018) Severity and burden of hand, foot and mouth disease in Asia: A modelling study. BMJ Glob Health 3: e000442. 
13. Zhao Y, Zhang H, Liu H, Sun H, Huang X, et al. (2017) Complete genome sequence of human echovirus 20 strain $812 / \mathrm{YN} / \mathrm{CHN} / 2010$, associated with severe hand-foot-and-mouth disease. Genome Announc 5: e00486.

14. Yao X, Bian LL, Lu WW, Li JX, Mao QY, et al. (2017) Epidemiological and etiological characteristics of herpangina and hand foot mouth diseases in Jiangsu, China, 2013-2014. Hum Vaccin Immunother 13: 823-830.

15. Li J, Sun Y, Du Y, Yan Y, Huo D, et al. (2016) Characterization of Coxsackievirus A6- and Enterovirus 71-Associated hand foot and mouth disease in Beijing, China, from 2013 to 2015. Front Microbiol 7: 391.

16. Li J, Pan H, Wang X, Zhu Q, Ge Y, et al. (2018) Epidemiological surveillance of hand, foot and mouth disease in Shanghai in 2014-2016, prior to the introduction of the enterovirus 71 vaccine Emerg Microbes Infect.7: 37.

17. Lin JY, Shih SR, Pan M, Li C, Lue CF, et al. (2009) hnRNP A1 interacts with the $5^{\prime}$ untranslated regions of enterovirus 71 and Sindbis virus RNA and is required for viral replication. J Virol 83: 6106-6114.

18. Too IH, Yeo H, Sessions OM, Yan B, Libau EA, et al. (2016) Enterovirus 71 infection of motor neuron-like NSC-34 cells undergoes a non-lytic exit pathway. Sci Rep 6: 36983.

19. Klionsky DJ, Schulman BA (2014) Dynamic regulation of macroautophagy by distinctive ubiquitin-like proteins. Nat Struct Mol Biol 21: 336-345.

20. Lee HR, Kim DH, Kim MG, Lee JS, Hwang JH, et al. (2016) The regulation of autophagy in porcine blastocysts: Regulation of PARylationmediated autophagy via mammalian target of rapamycin complex 1 (mTORC1) signaling. Biochem Biophys Res Commun 473: 899-906.

21. Chen Q, Yue F, Li W, Zou J, Xu T, et al. (2015) Potassium Bisperoxo(1,10phenanthroline)oxovanadate (bpV(phen)) induces apoptosis and pyroptosis and disrupts the P62-HDAC6 protein interaction to suppress the acetylated microtubule-dependent degradation of autophagosomes. J Biol Chem 290: 26051-26058.

22. Muhammad JS, Nanjo S, Ando T, Yamashita S, Maekita T, et al. (2017) Autophagy impairment by Helicobacter pylori-induced methylation silencing of MAP1LC3Av1 promotes gastric carcinogenesis. Int J Cancer 140: 2272-2283.

23. Frankel LB, Lund AH (2012) MicroRNA regulation of autophagy. Carcinogenesis 33: 2018-2025.

24. Chandra V, Bhagyaraj E, Parkesh R, Gupta P (2016) Transcription factors and cognate signalling cascades in the regulation of autophagy. Biol Rev Camb Philos Soc 91: 429-451.

25. Russell RC, Tian Y, Yuan H, Park HW, Chang YY, et al. (2013) ULK1 induces autophagy by phosphorylating Beclin-1 and activating VPS34 lipid kinase. Nat Cell Biol 15: 741-750.

26. Taylor MP, Burgon TB, Kirkegaard K, Jackson WT (2009) Role of microtubules in extracellular release of poliovirus. J Virol 83: 6599-6609.

27. Chan ST, Ou JJ (2017) Hepatitis c virus-induced autophagy and host innate immune response. Viruses 9: 224.

28. Dash S, Chava S, Aydin Y, Chandra PK, Ferraris P, et al. (2016) Hepatitis C Virus infection induces autophagy as a prosurvival mechanism to alleviate hepatic ER-stress response. Viruses 8: 150.

29. Lee YR, Kuo SH, Lin CY, Fu PJ, Lin YS, et al. (2018) Dengue virusinduced ER stress is required for autophagy activation, viral replication, and pathogenesis both in vitro and in vivo. Sci Rep 8: 489.

30. Datan E, Roy SG, Germain G, Zali N, McLean JE, et al. (2016) Dengueinduced autophagy, virus replication and protection from cell death require ER stress (PERK) pathway activation. Cell Death Dis 7: e2127e2127.

31. Kemball CC, Alirezaei M, Flynn CT, Wood MR, Harkins S, et al. (2010) Coxsackievirus infection induces autophagy-like vesicles and megaphagosomes in pancreatic acinar cells in vivo. J Virol 84: 12110-12124.

32. Wu H, Zhai X, Chen Y, Wang R, Lin L, et al. (2016) Protein 2B of Coxsackievirus B3 induces autophagy relying on its transmembrane hydrophobic sequences. Viruses 8: e131.
33. Fu Y, Xu W, Chen D, Feng C, Zhang L, et al. (2015) Enterovirus 71 induces autophagy by regulating has-miR-30a expression to promote viral replication. Antiviral Res 124: 43-53.

34. Hu DD, Mai JN, He LY, Li PQ, Chen WX, et al. (2017) Glucocorticoids prevent Enterovirus 71 capsid protein vp1 induced calreticulin surface exposure by alleviating neuronal ER stress. Neurotox Res 31: 204-217.

35. Chen D, Feng C, Tian X1, Zheng N, Wu Z (2018) Promyelocytic leukemia restricts Enterovirus 71 replication by inhibiting autophagy. Front Immunol 9: 1268

36. Sarkar C, Zhao Z, Aungst S, Sabirzhanov B, Faden AI, et al. (2014) Impaired autophagy flux is associated with neuronal cell death after traumatic brain injury. Autophagy 10: 2208-2222.

37. Li J, Liu Y, Wang Z, Liu K, Wang Y, et al. (2011) Subversion of cellular autophagy machinery by hepatitis B virus for viral envelopment. J Virol 85: 6319-6333.

38. O'Donnell V, Pacheco JM, LaRocco M, Burrage T, Jackson W, et al. (2011) Foot-and-mouth disease virus utilizes an autophagic pathway during viral replication. Virology 410: 142-50.

39. Killian MS (2012) Dual role of autophagy in HIV-1 replication and pathogenesis. AIDS Res Ther 9: 16.

40. Tsuganezawa K, Shinohara Y, Ogawa N, Tsuboi S, Okada N, et al. (2013) Two-colored fluorescence correlation spectroscopy screening for LC3P62 interaction inhibitors. J Biomol Screen 18: 1103-9.

41. Kuo RL, Kung SH, Hsu YY, Liu WT (2002) Infection with enterovirus 71 or expression of its $2 \mathrm{~A}$ protease induces apoptotic cell death. J Gen Virol 83: 1367-76.

42. Foo DG, Macary PA, Alonso S, Poh CL (2008) Identification of human CD4 T-cell epitopes on the VP1 capsid protein of enterovirus 71 . Viral Immunol 21: 215-24.

43. Victorio CBL, Xu Y, Ng Q, Meng T, Vincent TK, et al. (2016) Cooperative effect of the VP1 amino acids $98 \mathrm{E}, 145 \mathrm{~A}$ and $169 \mathrm{~F}$ in the productive infection of mouse cell lines by enterovirus 71 (BS strain). Emerg Microbes Infect 5: e60.

44. Xu J, Zhang C (2016) Human IgG Fc promotes expression, secretion and immunogenicity of enterovirus 71 VP1 protein. J Biomed Res 30: 209-216.

45. Yang CH, Li HC, Jiang JG, Hsu CF, Wang YJ, et al. (2010) Enterovirus type $712 \mathrm{~A}$ protease functions as a transcriptional activator in yeast. J Biomed Sci 17: 65.

46. Wang LC, Chen SO, Chang SP, Lee YP, Yu CK, et al. (2015) Enterovirus 71 Proteins $2 \mathrm{~A}$ and $3 \mathrm{D}$ antagonize the antiviral activity of gamma interferon via signaling attenuation. J Virol 89: 7028-37. 